\title{
Clinical Efficacy of an Osmotic, Antiviral and Anti-Inflammatory Polymeric Nasal Film to Treat Covid-19 Early-Phase Respiratory Symptoms
}

\author{
Remi Shrivastava' \\ Megha Vijay ${ }^{2}$ \\ Nathalie Maneby' \\ Ravishekhar Shrivastava $\mathbb{D}^{\prime}$ \\ IVITROBIO Research Institute, Issoire, \\ 63500, France; ${ }^{2}$ Mudra Clincare, Navi \\ Mumbai, India
}

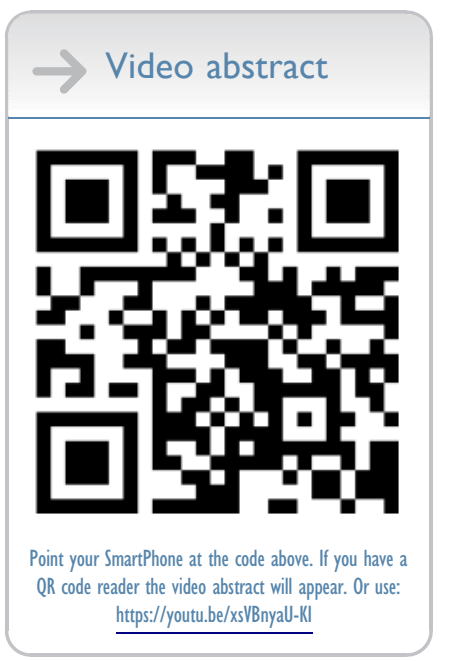

Correspondence: Ravishekhar Shrivastava Email rs@vitrobio.com
Background: Covid-19 infection is a multifactorial disease where the virus mainly enters through the nasal cavity, grows, triggers inflammation, and destroys nasal mucosa cells, allowing systemic virus entry and infection of other organs. When symptoms appear, the disease physiopathology is already established. The immune system tries to control the infection but if the infection persists, it gets tired, fatigued, stressed, and finally, there is burnout. Therefore, an effective treatment should be multi-targeted and should not focus only on one parameter. Minimizing virus concentration, reducing nasal mucosa inflammation, and keeping the nasal surface clean should lessen systemic infection and the probability of developing severe respiratory distress. We evaluated clinical efficacy and safety of an osmotic nasal surface cleaning, virus and cytokine trapping polymeric film, in early-stage Covid-19 positive symptomatic patients.

Methods: A randomized, multicentric, observational study was performed to evaluate the efficacy and safety of the osmotic film in 213 patients, randomized in 2 equal arms, and confined for 14-days just after the RT-PCR + test. Due to ethical reasons, all patients received symptomatic treatments (ST). In addition, the patients in Arm-1 received the test product (ST+TP, 2-3 sprays, 4-5 times/ day) for 14-days. Leicester Cough Questionnaire, Visual Analogue Scale, and Covid-19-associated symptoms such as fever, pain, taste, smell, and headache were evaluated daily. Home-confined patients were immediately hospitalized in case of aggravation of any life-threatening clinical sign. Results: Among 213 patients, 98 in the ST and 102 in the ST+TP group completed the study. Thirteen patients did not complete the study as 8 in the ST group and 5 in the ST+TP group were hospitalized during the study due to low blood oxygen levels or sudden health deterioration and were excluded from the study. In the remaining control standard treatment group ( $n=98)$, clinical signs sharply aggravated on day 1-2 followed by stabilisation between days 3-6 and progressive reduction thereafter. In the test product group $(n=102)$, symptoms were stabilized just after the first application and improved progressively. Only 42/102 patients in the test product group presented nasal discharge or irritation due to the osmotic properties of the product.

Conclusion: The continuous multi-target approach of detaching and neutralizing virus particles and pro-inflammatory cytokines from the nasal surface to minimize systemic virus exposure is a very logical and efficient approach to avoid Covid-19-induced systemic pathology.

Keywords: clinical trial, Covid-19, osmotic filmogen barrier

\section{Introduction}

Covid-19 is a highly contagious disease (COVID-19) caused by Coronavirus 2 and responsible for Severe Acute Respiratory Syndrome (SARS-CoV-2S). ${ }^{1,2}$ Nearly 90\% of infections occur by means of virus inhalation when the virus spike (S) protein 
encounters angiotensin-converting enzyme 2 (ACE2) receptors on nasal mucosa (NM). ${ }^{2,3}$ The Receptor-Binding Domain (RBD) of $\mathrm{S}$ protein allows virus fusion with the host cell membrane. ${ }^{2,4}$

Like in many other airborne viral diseases, nasal mucosa is concerned by the first step of the infection as it contains the highest levels of ACE2 and of the transmembrane protease, serine- 2 receptors. ${ }^{5}$ These receptors act as main entry gates for virus infection. $^{6-8}$ Once it has entered the cells, the virus multiplies and elicits a highly inflammatory form of programmed cell death termed pyroptosis, which activates innate immune cells and releases Covid-19 disease-specific interleukin-6, TNF-alpha and other proinflammatory cytokines. ${ }^{9-11}$ In most cases, the immune system neutralizes the virus within 6-7 days and the patient starts recovering, but in certain cases, the body's defenses are too stressed to control virus growth which leads to a "cytokine storm". ${ }^{12,13}$ Severe inflammation in the upper and lower respiratory tracts may have fatal consequences. Disease severity and symptoms may vary considerably from patient to patient, but the main symptoms include respiratory difficulties, cough, fever, fatigue, loss of taste and smell with diarrhea or myalgia in a few cases. $^{1,14}$ In most cases, the immune system neutralizes the virus within 6-7 days and the patient starts recovering, but in certain cases, the body's defenses are too stressed to control virus growth which leads to fatal consequences. The main reason behind this sudden and severe systemic pathogenesis is not yet clear as in addition to the severe pulmonary "cytokine storm",12,13 a novel hematological syndrome manifested as vascular endothelial damage, complement activation, and thrombosis, seriously compromising circulatory functions is also gaining strong acceptance ${ }^{15}$ Vascular damage leading to pulmonary hypertension, chronic obstructive pulmonary disease, congestive heart failure, myocardial injury, and pulmonary embolism is postulated to generate respiratory syndrome. ${ }^{16}$ Other pathological or malignant conditions, not affecting pulmonary functions, do not appear to affect Covid-19 prompted mortalities. $^{17}$

Just after virus infection in the nasal cavity, virus multiplies vigorously in the nasal mucosa, destroys cells and liberates huge quantities of free virus particles on the nasal surface. This triggers further release of pro-inflammatory cytokines and associated cellular damage. ${ }^{18}$ Damaged and inflamed nasal mucosa cannot play its role as a natural barrier, facilitating virus entry into the circulation and the devastating consequences. Therefore, stopping or minimizing virus growth and nasal mucosa inflammation to reduce systemic infection and immune stress should constitute the best therapeutic approach, but there is no topical antiviral, nasal surface cleaner or cell growth stimulator drugs yet available. ${ }^{19,20}$
Saline or salt-containing nasal washes are not effective because such treatments are diluted immediately with the osmotic liquid flow and get disintegrated rapidly. The same is true for highly osmotic solutions such as glycerol, which may generate higher osmotic flow to detach and drain free floating virus and cytokine particles from the nasal mucosa, but the higher osmotic force generated by such solutions causes strong irritation and leads to rapid film disintegration.

Glycerol being a highly osmotic and cell-friendly solution, our aim was to conceive an osmotically active but stable glycerol film for long-lasting nasal surface retention. To stabilize the glycerol film for 4-6h while preserving its osmotic potential, we added a very small quantity $(<0.70 \%)$ of specific glycerol molecule-binding polymers or plant tannins in the solution. Tannins are very big and inert molecules with an equally strong capacity to bind with selected proteins. We therefore selected those dual acting tannins which can bind with glycerol molecules, on the one hand, and with specific Covid-19 disease-specific proteins, on the other hand. ${ }^{21}$ The affinity of polymers to bind with glycerol molecules $(100 \%)$ as well as with the Covid-19 disease-specific proteins (80-90\%) was evaluated using ELISA tests. The resultant stable filmogen solution was rendered non-irritant by incorporating appropriate concentrations of thickeningjellifying agents which swell and render the film absorbent, when in contact with water.

It was hypothesized that nasal surface application of such a mechanical pressure-resistant, absorbent, and osmotic film containing specific protein molecule-binding polymers, should generate sufficient continuous osmotic flow to detach and drain the proteins towards the film where they can be captured through polymeric binding. Cleaning the nasal mucosa through mechanical action should minimize the concentration of viruses and inflammatory cytokines on the nasal surface and in consequence, should minimize systemic infection.

The clinical efficacy and safety of this polymeric osmotic film were evaluated in Covid-19 positive patients during the initial phase of infection.

\section{Study Design}

This was a randomized, multicentric, observational study to evaluate the efficacy and safety of the test product (Code: PIRDAL, Common name: Covispray) in preventing symptomatic manifestation of disease in Covid-19 proven cases. The study design and its flowchart are presented in Figure 1. 


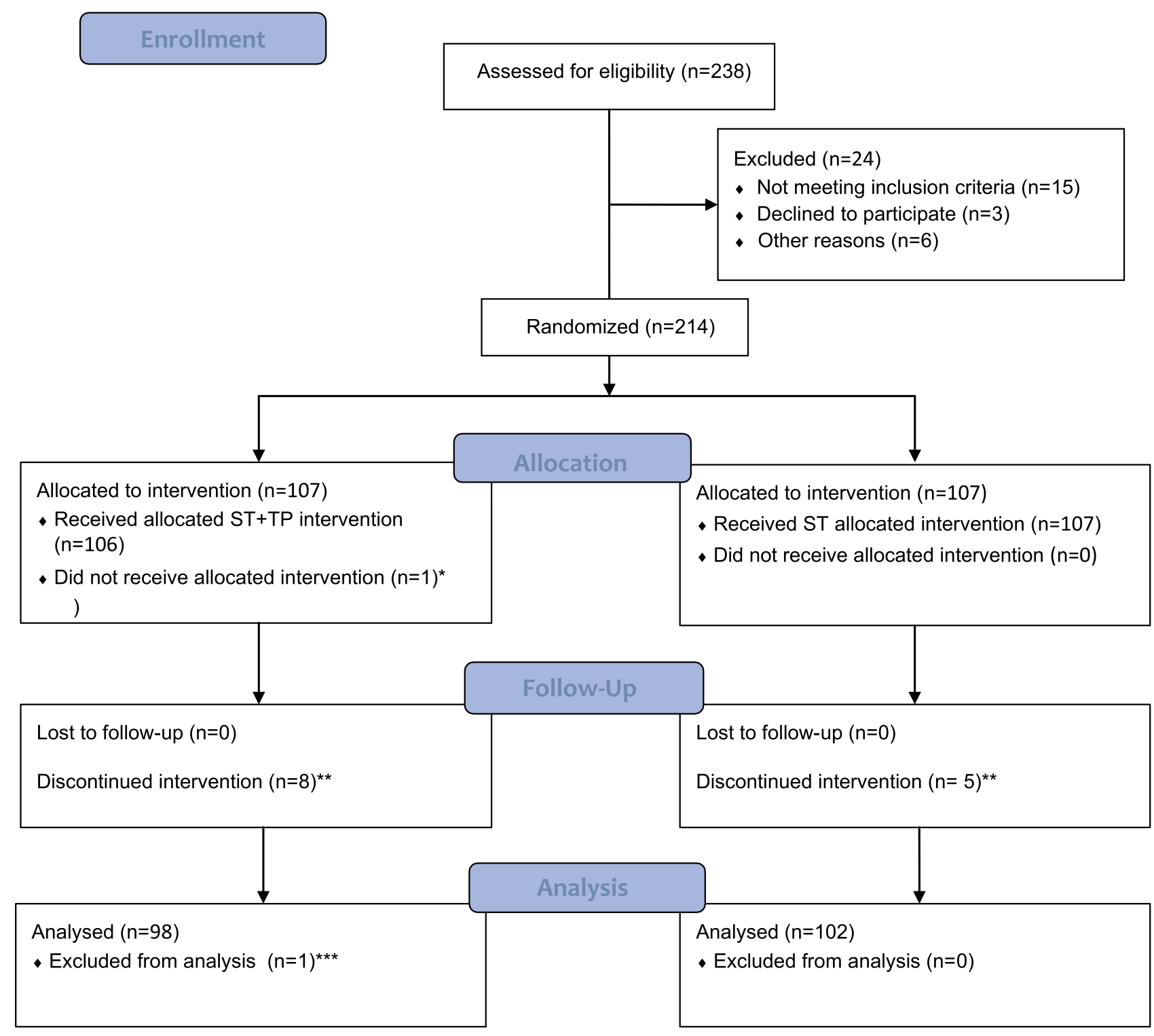

Figure I Consort flow diagram. *Dropped out after randomization due to increasing clinical signs before first treatment, **hospitalized for intensive care, ***dropped out before first treatment.

\section{Trial Oversight}

The study was sponsored by VITROBIO France and was performed by MUDRA CLINCARE, Koparkhairane, Navi Mumbai-400709, India. The protocol was approved by relevant ethics committees (Altezza Institutional Ethics Committee, Shree Ashirwad Hospital, Dombivli, Maharashtra, India) and institutional review boards. The trial was registered on 26/11/2020 under $\mathrm{n}^{\circ}: \mathrm{CTRI} / 2020 / 11 / 029388$ (http://ctri.nic.in). The authors vouch for the conduct of the trial, adherence to the protocol, the accuracy and completeness of the data and analyses, and the reporting of adverse events. The trial complied with the International Conference on Harmonisation Guidelines for Good Clinical Practice, the principles of the Declaration of Helsinki, and relevant national and local regulations. At the time of screening, participants signed written informed consent. The sponsor provided the trial medication and supplied relevant product investigational information. An independent organization, RAJ Consultancy (Plot 61, Sector 10, Kamothe, Navi Mumbai, India) was commissioned to audit the study. All the patients were recruited in Mumbai (India) and its vicinity as soon as the Maharashtra government Covid-19 follow-up medical organisations decided to home confine the patient following positive PCR test. Clinical trial institute worked in collaboration with these organisations. The first patient was recruited at the end of November 2020 and the last patient followed was in 
January 2021.The study data were submitted to Soladis Statistics (Lyon, France) for statistical analyses.

\section{Key Inclusion and Exclusion Criteria}

Main inclusion criteria were (1) Male or female, not pregnant or lactating, aged between 18-70 years, (2) Just detected positive for Covid-19 by RT-PCR test, (3) Had slight to moderate initial nasal/respiratory Covid-19 symptoms, (4) Accepting to be confined 14-days under the supervision of Indian medical authorities with or without other family members, (5) Accepting not to self-medicate except for the symptomatic medications which may be prescribed by their physician, (6) ready to give written informed consent, (7) Ready to follow the protocol and fill-up the daily diary/on-line questionnaire, (8) Not under any antiviral treatment, (9) Not suffering from any chronic disease which may impact study parameters, (10) Not suffering from severe diabetes or hypertension.

The main non-inclusion criteria were (1) Not meeting any of the above inclusion criteria (2) Patients having severe Covid-19 related clinical signs, (3) Patients having evidence of immunosuppression, or under any antiviral treatment, or detected positive for serum antibodies against Covid-19, (4) Allergy to any of the investigational product ingredients, (5) Recent nasal surgery or abnormal structural narrowing of sinus passages, any medical condition within the last 6 months which may affect study objectives.

Patients showing serious illness were not included in the study due to ethical reasons as they would have required rapid medical assistance. Taking into consideration the mode of action of the test product, patients having strong respiratory symptoms or chronic Covid-19 infection were not included. Hypertension and diabetic being common diseases in India, only severely affected patients were excluded.

\section{Trial Endpoints}

The primary endpoints were the mean change in the intensity of cough respiratory symptoms assessed using the widely accepted and validated Leicester Cough Questionnaire (LCQ), ${ }^{22,23}$ between the two groups from the baseline (day 0), daily during the fourteen days of treatment. The patients recorded their answers to the standard LCQ 19 cough-based questions daily.

Visual analogue scale (VAS) includes self-evaluation by the patient of the cough respiratory symptoms on a 0 to 10 scale during the fourteen days of treatment. ${ }^{24,25}$ VAS quantifies whether a person had a cough, difficulties while coughing, work performance, and cough-related discomfort. Total mean values in each group are compared. Other daily general symptom scoring included fever, loss of taste, change in smell, headache, body aches, body pain, sensation of weakness, sore throat, eye irritation, day and night cough frequency, nausea, blood oxygen saturation, as well as all adverse events and in-study hospitalisation, in both groups. The mean result outcome was compared to baseline at each time point, for each group, from the daily questionnaire filled out by each patient during the 14-day trial period.

Due to ethical reasons, patients showing any lifethreatening clinical signs, whether respiratory-related or poor blood oxygen level, were hospitalized for intensive care and were removed from the study. In the absence of any specific treatment against Covid-19, the ethics committee obliged allowing minimum use of any symptomatic treatment (eg, analgesics, anti-inflammatory, and ayurvedic plant preparations except antivirals) prescribed by their physician for all the patients during the study period.

\section{Randomization}

Patients satisfying all inclusion criteria were enrolled and randomly allocated in a 1:1 ratio as per the randomization schedule. Randomization was performed using SAS Version 9.1.3. The randomization schedule was generated with block randomization methodology. Patients were randomized in 2-Arms. Arm 1: Patients received any symptomatic treatment (ST) and the test product (TP) while the Arm-2 control patients were given only ST.

\section{Treatment}

As per the randomization, patients in the ST+TP group received three $15-\mathrm{mL}$ nasal spray containers with instructions to apply 2-3 sprays in each nostril, 4-5 times per day for 14 consecutive days. The nasal sprays were supplied by VITROBIO SAS, France. The solution contained glycerol which was rendered filmogen with a specific association of dual acting (glycerol + specific protein binding) S1-cyanidins derived from plant extracts of Tanacetum parthenium, Curcuma longa and Urtica dioica, and Camellia sinensis, with food-grade preservatives (Sodium benzoate, Potassium sorbate, Citric Acid), qsp water.

\section{Statistical Analyses}

Estimations based on nasal sprays with similar compositions for rhinosinusitis ${ }^{26}$ and allergic rhinitis ${ }^{18,27}$ predicted that a sample of 75 analysed patients in each group would provide $90 \%$ power to detect data significance. With an anticipated rate of discontinuation of $40 \%, 220$ participants in total were 
planned for randomization in this trial. Analyses were conducted in the modified intention-to-treat population, which included all randomly assigned patients who received at least one dose of a trial regimen. The primary efficacy outcome was analysed using a parametric mixed model for repeated measures. For each day, the model estimated mean and its associated standard error $( \pm \mathrm{SE})$ are presented for each treatment group. The model estimated mean difference between groups and its $95 \%$ confidence interval $(\mathrm{CI})$ are also presented for each day. $P<0.05$ was considered statistically significant. Adverse event data were collected during the intervention period. The analyses were carried out with the software SAS v9.4.

\section{Results}

Dropouts: Of the 214 patients recruited at randomization, 98 in the ST and 102 in the ST+TP group completed the study. Among the 14 dropouts, 1 patient was excluded on day 1 before treatment due to increased symptom severity. In total 13 patients were hospitalized during the study due to low blood oxygen levels and sudden aggravation of one of the respiratory symptoms between days 6 to 11 of treatment. Among the 13 hospitalized patients, 8 were from ST group (hospitalized on day 6, 7, 8, 8, 9, 9, 10,11 ) and 5 from the ST+TP group (hospitalized on day $6,8,8,9,9)$. They were excluded from the study.

\section{Primary Endpoints}

LCQ: Patients rated their condition daily for the questionnaire according to the frequency of occurrence on a 1 to 7 scale where 1 represents severe cough symptoms and 7 the least. The mean LCQ scores in each group on each day are presented in Figure 2.

As shown in Figure 2, the mean total rating of 17 individual scores in the study varied between 92.5 and 117.7 (mean for 17 parameters between 5.44 and 6.92) during the trial. At randomisation (day 0 ) the mean of total LCQ scores was $103.4 \pm 0.77$ in ST group $(\mathrm{n}=106)$ and $104.5 \pm 0.77(\mathrm{n}=107)$ in the $\mathrm{ST}+\mathrm{TP}$ group (NS on day 0$)$.

In the ST group, on day 1, the mean coughing symptoms LCQ score aggravated sharply (mean reduction of $-6.3)$ which continued worsening in severity on day 2 $(-10.9$ vs day 0$)$. The severity of cough remained identical or worsened very slightly between days $3-5$ but the symptoms started improving thereafter, slowly, and progressively, up to the end of the study.

Surprisingly, in the ST+TP group there was no increase in mean LCQ scores up to day 3. Symptom severity remained stable during these days (mean score $104.5 \pm$ 0.77 on day 0 and 105.4 on day $3 \pm 0.57$ ). From day 4 onwards, the mean cough symptom intensity started improving, slowly and progressively, up to day 14 (111.5 \pm 0.42 on day $7 ; 116.2 \pm 0.38$ on day 10 ; and $117.8 \pm 0.50$ on day 14). Due to the stabilization of clinical symptoms right after the first treatment in the ST $+\mathrm{TP}$ group during the first 3 days, the difference with ST group was highly statistically significant $(p<0.001)$ during the entire study period. These results show the Covid-19-induced cough symptoms aggravate very fast during the first 3 days, but the TP treatment blocks worsening of the symptoms immediately after the first application.

\section{VAS Outcome}

At the start of the study, the totals of all mean VAS scores were $1800.2 \pm 60.61$ in the ST and $1723.5 \pm 60.33$ in the ST + TP groups (NS). In the ST group, the mean score continued rising to day $5(2319.1 \pm 60.611)$ but started decreasing thereafter slowly and progressively with a mean of 2135.60 ( \pm $60.84)$ on day 7 and $1200.40( \pm 62.94)$ on day 14. In the ST + TP group, the score remained nearly stable up to day 3 $(1769.10 \pm 60.33)$ and started decreasing rapidly thereafter with scores of $1288.6 \pm 60.47$ on day 7 and only $415.7 \pm 61.66$ on day 14 ( $<00.001$ from day 1 onwards; Figure 3). These results show that Covispray treatment significantly reduced cough-related parameters in a slow and progressive fashion confirmed by the LCQ outcome.

\section{Effect on Covid-19 Induced Concomitant Symptoms \\ Fever (Figure 4A)}

The number of patients having fever at BL and on day 1 was nearly identical in both groups (about 65\% of patients). From day 2 onwards, fever progressively decreased in both groups, but the reduction was much faster in the ST+TP group with only 43/102 patients on day $3,26 / 102$ on day $4,14 / 102$ on day 5 , only $1 / 102$ on day 10 and none from day 11 onwards. In the control group, 34/98 patients had fever on day 7 but the incidence reduced slowly with $11 / 98$ patients on day 10 and only $2 /$ 98 on day 14. These results show that cleaning the nasal surface contaminants at the start of the disease dramatically reduces the incidence of fever.

\section{Effect on Loss of Smell (Figure 4B)}

Only 40/102 patients in the ST+TP group complained of loss of sense of smell compared to 53/106 in the control 


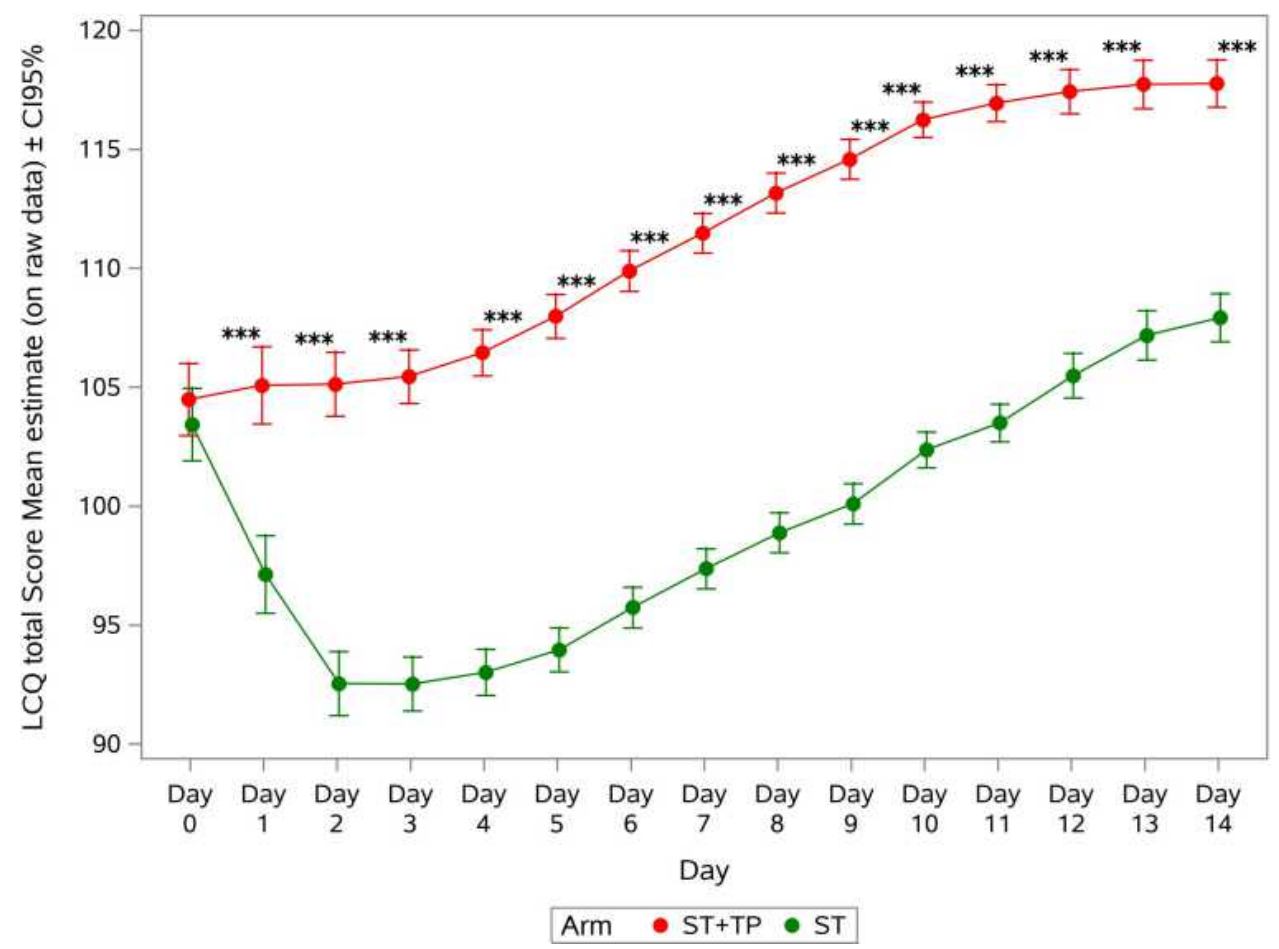

Figure 2 LCQ total Score (on each day) mean estimate (on raw data) \pm C195\% in the ST+TP group ( $n=107)$ and in the ST group ( $n=106)$. Statistical analysis was performed using a mixed model for repeated measurements on raw data. $\mathrm{P}<0.00 \mathrm{I}^{* * *}$.

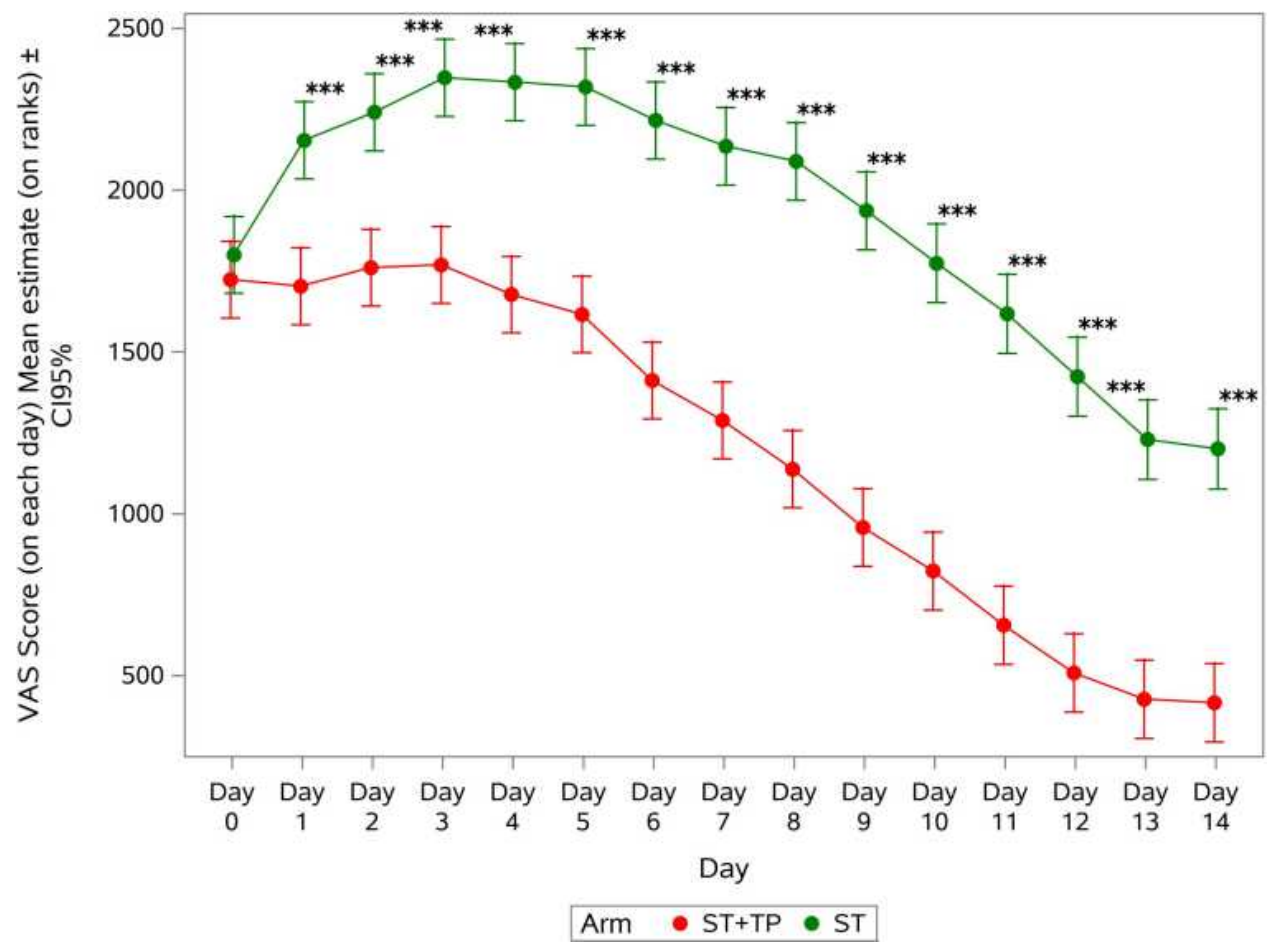

Figure 3 VAS Score (on each day) mean estimate (on ranks) $\pm C 195 \%$ in the ST $+T P(n=107)$ and in the ST $(n=106)$ group. Statistical analysis was performed using a mixed model for repeated measurements on raw data transformed into ranks. $P<0.001^{* * *}$. 


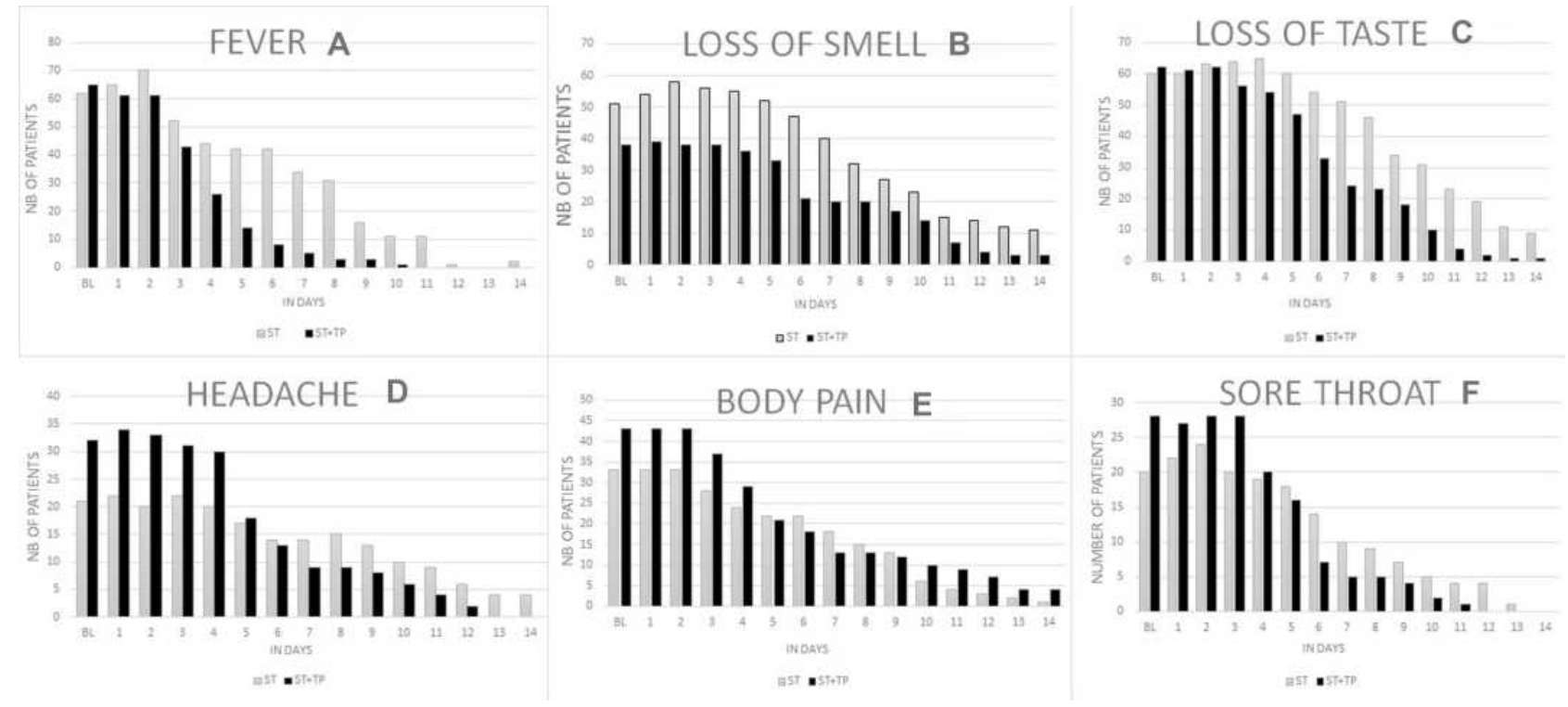

Figure 4 Number of patients affected by fever $(\mathbf{A})$, loss of smell $(\mathbf{B})$, loss of taste $(\mathbf{C})$, headache (D), body pain (E), and sore throat (F) on each day in each group. Gray bars represent ST group $(n=98)$ and black bars ST+TP group $(n=102)$.

ST group at the time of recruitment. This shows that Covid-19 + patients suffer less from loss of smell than the loss of taste. Smell sensation started improving in both groups only after day 5 but the reduction was slightly better in the ST+TP group $(\mathrm{p}<0.05$ between days 2 to 7 and from day 12).

\section{Loss of Sense of Taste (Figure 4C)}

This is an important clinical sign in Covid-19 infected patients as $38.7 \%$ patients in ST $(41 / 106)$ and $40.2 \%$ in $\mathrm{ST}+\mathrm{TP}(43 / 107)$ group noticed change in taste at baseline. The Covispray treatment had no effect on restoring loss of taste during the first 2 days of treatment compared to the baseline values, but a progressive recovery was observed, particularly from day 5 onwards. Only $25 \%$ patients complained of taste loss on day $7,10 \%$ on day 10 , and only $1 \%$ on day 14 . In the ST group, the loss of taste remained unchanged during the first 5 days (39/106 between days 0 to 5) with a progressive recovery of taste from day 6 onwards (32/98 on day $10,9 / 98$ on day $14 ; \mathrm{p}<0.05$ vs $\mathrm{ST}+\mathrm{TP}$ from day 4 onwards). The recovery of taste loss was faster in ST+TP group, which is concomitant with the improvements in LCQ, VAS, and fever observed in this group.

\section{Effect on Headache (Figure 4D)}

Only 21 patients in the ST group complained of headache compared to 32 in the ST+TP group at the start of the study. In both groups, the incidence of headache started decreasing only after day 4 but the reduction in the ST+TP group was much faster compared to the ST group patients from day 5 and no patient complained of headache from day 12 onwards. In the ST group, the reduction was progressive and 4/21 patients still continued to have headache on day 14. These results show that the beneficial effects of TP during the first 4 days of treatment equally affect all other collateral health parameters simultaneously.

\section{Effect on Body Aches and Pain (Figure 4E)}

The number of patients having body aches and pain was nearly $40 \%$ higher in the ST+TP group at baseline compared to the ST group, with no change in this parameter up to day 3 in both groups. The incidence started decreasing in both groups from the third day of treatment, but the improvement appeared slightly better in the ST+TP group. It is concluded that there is no significant difference between the two groups with respect to improvement on body aches and pain. This may have been related to the fact that the virus continues residing inside the body and generating inflammation which is not much affected by cleaning the contaminants only from the nasal surface.

\section{Effect on Sore Throat (Figure 4F)}

At BL, the incidence of sore throat was observed to be 20/ 98 patients in the ST group and 28/102 patients in the ST $+\mathrm{TP}$ group. There was no change in this parameter in both groups up to day 3 but from day 4 onwards there was a drastic reduction of sore throat in the ST+TP group with 
only $7 / 28$ patients with sore throat on day 6,2 on day 10 and 0 on day $12 \mathrm{v} / \mathrm{s} 14 / 20$ patients on day $6,10 / 20$ on day $10,4 / 20$ on day 12 in the ST group. No patient complained of sore throat in either group on day 14.

\section{Day and Night Cough Frequency}

Identical to the effects observed in VAS, the day and night cough frequencies were also proportionally decreased after 4-5 days of treatment in the ST+TP group compared to the ST group.

There were no significant differences between the two groups for other parameters including blood $\mathrm{O} 2$ saturation level, respiratory rate, blood pressure, heart rate, pulse rate, nausea, and eye irritation.

\section{Secondary Parameter - Adverse Events}

There was no moderate or severe adverse event recorded in either group during the study. 13 patients (8 in ST and 5 in ST+TP) dropped out because their blood O2 level decreased or some of the Covid-19 clinical symptoms deteriorated in these patients. There was no mild adverse event noticed in any of the ST group patients, but $42 / 102$ patients in the Covispray (ST+TP) group had mild adverse effects related to the application of the test product. The main events were (1) nasal irritation or nasal discharge, usually during the first few minutes just after the product application, and (2) watering of the eyes after product use in as many as $42 / 102$ patients. These effects are considered to be related to the product because nasal mucosa osmosis starts immediately after product application which attracts hypotonic liquid from the nasal surface. This sudden reduction of intracellular liquid is felt as mild to moderate irritation which may even lead to eye discharge. The cellular liquid loss is immediately (within $1 \mathrm{~min}$ ) replaced by circulating liquid which stops further feelings of irritation. As this event is related to the mode of action of the Covispray hypertonic filmogen osmotic solution, and as it has no residual adverse consequences, these effects are considered not harmful to the patient.

One case of nausea and one of vomiting are isolated cases and are common in Covid-19 symptomatic patients. Therefore, they are not considered as related to the product.

\section{Discussion}

Multiple studies clearly show that the nasal cavity is the primary route of infection and transmission of Covid-19 virus. ${ }^{6,28}$ The very high concentration of ACE2 receptors on the inner lining of nasal mucosa favours virus growth in the nasal cavity. ${ }^{6,28}$ The virus grows and destroys these cells, triggers an inflammatory cascade with the liberation of multiple pro-inflammatory cytokines, minimizes the natural barrier and defensive functions of nasal mucosa, enters the circulation, and infects cells in other tissues which may lead to serious illness. ${ }^{28}$ This is the reason why nasal irrigation, also called nasal lavage, with saline or salt solutions, with or without other antimicrobial agents, is suggested in order to reduce the systemic consequences of Covid-19 infection. ${ }^{20,29}$ Such treatments are poorly effective as they disintegrate rapidly and have a short duration of action. We used a highly osmotic, dual acting polymer containing a stable glycerol film to detach and drain but also to trap incoming virus particles, cytokines, and other free-floating contaminants from the nasal surface. ${ }^{30}$ The filmogen polymers were selected based on their strong affinity for viral S1 and RBD proteins as well as IL-6 and TNF-alpha Covid-19 specific proinflammatory cytokines.

Covid-19 infected patients normally show respiratory symptoms, which aggravate during the first 4-6 days due to virus growth, inflammation, and nasal mucosa damage. ${ }^{31}$ Clinical trial results in ST group patients equally show a strong aggravation of the mean respiratory symptoms (LCQ and VAS parameters) on days 1 and 2 followed by further slow deterioration up to day 4-6 and progressive improvement thereafter. In the $\mathrm{ST}+\mathrm{TP}$ group, the respiratory symptoms did not aggravate on days 1 and 2 but remained stable up to day 5 followed by slow improvement, parallel to the ST group, up to day 14. The mean LCQ and VAS score difference between the TP and the ST groups was statistically significant from day 1 onwards. The reason for this stabilization of respiratory symptoms immediately after the first treatment probably resides in the fact that the TP acts instantly by cleaning newly generated virus particles as well as inflammatory cytokines towards the film where they are trapped and inactivated by polymers. This mechanical cleaning minimizes cellular destruction and stimulates reconstruction of nasal mucosa. ${ }^{28,29}$ TP cleans only the free-floating nasal surface virus and protein molecules but has no effect on the intracellular and circulating virus particles which continue pouring onto the nasal surface and maintaining the infection for a few weeks. ${ }^{19}$ This explains why there is a sharp drop of Covid-19 clinical symptoms during the first 4-6 days, but further improvement is progressive and slow. Our objective was not to cure the disease but simply 
to minimize the amount of virus and the inflammatory proteins on the nasal surface to reduce circulating virus concentration, immune stress, and the chances of severe infection in the lower respiratory tract. This approach minimizes the probability of developing severe respiratory symptoms with a simple and safe device.

The main site of Covid-19 virus mutation is $\mathrm{S} 1$ protein $^{32}$ The polymers incorporated in Covispray are selected to bind with whole COVID-19 S1 proteins as described in details by $\mathrm{R}$ Shrivastava et al. ${ }^{33}$ As viral mutations occur at the level of S1 protein, which may impact virus infectivity, antigenicity and immunogenic profile of the vaccines, ${ }^{34}$ Covispray should be effective against all the covid-19 variants. ${ }^{30}$

A Small Video attached with this article, explains the mode of action and the efficacy of Covispray.

Except for slight nasal irritation and accelerated nasal discharge during the first 5-10 minutes, no drug-related side effects were detected in any of the patients during the trial. This is understandable because the product acts mechanically as a filmogen barrier on the nasal mucosa without any interactions with the underlying cells. The barrier also protects the nasal surface from incoming environmental virus particles and other contaminants.

This mechanical approach of preventing new viral infection and minimizing the concentration of free virus particles as well as Covid-19 specific cytokines from the nasal surface may not cure the disease but may help to attenuate early-phase clinical symptoms and to minimize the occurrence of severe respiratory distress, irrespective of the Covid-19 strain responsible for triggering the infection.

\section{Abbreviations}

COVID-19, coronavirus disease 2019; SARS-CoV-2, severe acute respiratory syndrome coronavirus 2; S1cyanidins, specific protein-binding association of polymers; ACE2, angiotensin-converting enzyme 2; ST, standard treatment; TP, test product; LCQ, Leicester cough questionnaire.

\section{Data Sharing Statement}

The data presented in this study are available on request from the corresponding author.

\section{Author Contributions}

All authors made a significant contribution to the work reported, whether that is in the conception, study design, execution, acquisition of data, analysis and interpretation, or in all these areas; took part in drafting, revising or critically reviewing the article; gave final approval of the version to be published; have agreed on the journal to which the article has been submitted; and agree to be accountable for all aspects of the work.

\section{Funding}

This research received no external funding and was entirely financed by VITROBIO Pharma Research Institute in France.

\section{Disclosure}

Dr Megha Vijay reports non-financial support from Mudra Clincare, during the conduct of the study; non-financial support from Mudra Clincare, outside the submitted work. The authors declare no conflict of interest related to this work.

\section{References}

1. World Health Organization. Coronavirus disease (COVID-19); 2021. Available from: www.who.int/emergencies/diseases/novelcoronavirus-2019. Accessed January 30, 2021.

2. Shi Y, Wang G, Cai XP, et al. An overview of COVID-19. J Zhejiang Univ Sci. 2020;21:343-360. doi:10.1631/jzus.B2000083

3. Zhang H, Penninger JM, Li Y, et al. Angiotensin-converting enzyme 2 (ACE2) as a SARS-CoV-2 receptor: molecular mechanisms and potential therapeutic target. Intensive Care Med. 2020;46:586-590. doi:10.1007/s00134-020-05985-9

4. Benton DJ, Wrobel AG, Xu P, et al. Receptor binding and priming of the spike protein of SARS-CoV-2 for membrane fusion. Nature. 2020;588:327-330. doi:10.1038/s41586-020-2772-0

5. Zou L, Ruan F, Huang H, et al. SARS-CoV-2 viral load in upper respiratory specimens of infected patients. $N$ Engl J Med. 2020;382 (12):1177-1179. doi:10.1056/NEJMc2001737

6. Sungnak W, Huang N, Bécavin C, et al. SARS-CoV-2 entry factors are highly expressed in nasal epithelial cells together with innate immune genes. Nat Med. 2020;26:681-687. doi:10.1038/s41591020-0868-6

7. Hou YJ, Okuda K, Edwards CE, et al. SARS-CoV-2 reverse genetics reveals a variable infection gradient in the respiratory tract. Cell. 2020;182(2):429-446. doi:10.1016/j.cell.2020.05.042

8. Ziegler CG, Allons SJ, Nyquist SK, et al. SARS-CoV-2 receptor ACE2 is an interferon-stimulated gene in human airway epithelial cells and is detected in specific cell subsets across tissues. Cell. 2020;181(5):1016-1035. doi:10.1016/j.cell.2020.04.035

9. Raewyn GC. SARS-CoV-2 and the nose: risks and implications for primary care. AJGP. 2020;49. doi:10.31128/AJGP-05-20-5452

10. Yap JK, Moriyama M, Iwasaki A. Inflammasomes and Pyroptosis as Therapeutic Targets for COVID-19. J Immunol. 2020;205 (2):307-312. doi:10.4049/jimmunol.2000513

11. Rousse M, Cucuat N, Janicot C, et al. Innovative scientific concept of topical virus glycoprotein inhibitors incorporated in hyperosmotic glycerol revolutionizes future prospects in the treatment of viral and bacterial throat infections. Int J Pharm Sci Drug Res. 2014;6 (1):01-11.

12. Shimabukuro-Vornhagen A, Gödel P, Subklewe M, et al. Cytokine release syndrome. J Immunol Ther Cancer. 2018;6:56. doi:10.1186/ s40425-018-0343-9 
13. Ragab D, Salah Eldin H, Taeimah M, et al. The COVID-19 cytokine storm; what we know so far. Front Immunol. 2020;11:1446. doi:10.3389/fimmu.2020.01446

14. Lechien JR, Chiesa-Estomba CM, Place S, et al. Clinical and epidemiological characteristics of 1420 European patients with mild-tomoderate coronavirus disease 2019. J Intern Med. 2020;288 (3):335-344. doi:10.1111/joim.13089

15. Wang X, Sahu KK, Cerny J. Coagulopathy, endothelial dysfunction, thrombotic microangiopathy and complement activation: potential role of complement system inhibition in COVID-19 [published online ahead of print, 2020 Oct 15]. J Thromb Thrombolysis. 2020:1-6.

16. Khusid JA, Becerra AZ, Gallante B, et al. Cancer, mortality, and acute kidney injury among hospitalized patients with SARS-CoV-2 infection. Asian Pac J Cancer Prev. 2021;22(2):517-522. doi:10.31557/APJCP.2021.22.2.517

17. Mishra A, Lal A, Sahu KK, George AA, Martin K, Sargent J. An update on pulmonary hypertension in Coronavirus Disease-19 (COVID-19). Acta Biomed. 2020;91(4):e2020155. PMID: 33525228; PMCID: PMC7927547. doi:10.23750/abm.v91i4.10698

18. Shrivastava L, Schütte H, Malik $\mathrm{P}$, et al. A new class of polymeric anti-allergen nasal barrier film solution for the treatment of allergic rhinitis. J Allergy Ther. 2017;8:3. doi:10.4172/2155-6121.1000263

19. Shrivastava RM, Tourret E, Schutte H, et al. Clinical efficacy of a new filmogen polymeric glycerol solution for the treatment of rhinosinusitis. J Clin Respir Dis Care. 2017;3:130.

20. Farrell NF, Klatt-Cromwell C, Schneider JS. Benefits and safety of nasal saline irrigations in a pandemic - washing COVID-19 away. JAMA Otolaryngol Head Neck Surg. 2020;146(9):787-788. doi:10.1001/jamaoto.2020.1622

21. Shrivastava RM, Shrivastava L, Shrivastava R Composition for topical application comprising glycerol and tannins. Patent PCT/EP2013/ 061835. EU. 2013.

22. Yousaf N, Lee KK, Jayaraman B, et al. The assessment of quality of life in acute cough with the Leicester Cough Questionnaire (LCQ-acute). Cough. 2011:7. doi:10.1186/1745-9974-7-4

23. Spinou A, Birring SS. An update on measurement and monitoring of cough: what are the important study endpoints? J Thorac Dis. 2014;7:S728-S734.
24. Leconte S, Ferrant D, Dory V, et al. Validated methods of cough assessment: a systematic review of the literature. Respiration. 2011;81:161-174. doi:10.1159/000321231

25. Altenburg J, Wortel K, De Graaff CS, et al. Validation of a visual analogue score (LRTI-VAS) in non-CF bronchiectasis. Clin Respir J. 2016;10(2):168-175. doi:10.1111/crj.12198

26. Shrivastava R, Deshmukh S, Rousse M. Comparison of a hypertonic tannin-rich solution vs $3 \% \mathrm{NaCl}$ solution as treatment for rhinosinusitis. Semant Scholar. 2013.

27. Greiner AN, Hellings PW, Rotiroti G, et al. Allergic rhinitis. Lancet. 2012;378(9809)

28. Gallo O, Locatello LG, Mazzoni A, et al. The central role of the nasal microenvironment in the transmission, modulation, and clinical progression of SARS-CoV-2 infection. Mucosal Immunol. 2020. doi:10.1038/s41385-020-00359-2

29. Casale M, Rinaldi V, Sabatino L, et al. Could nasal irrigation and oral rinse reduce the risk for COVID-19 infection? Int J Immunopathol Pharmacol. 2020;34:205873842094175.

doi:10.1177/ 2058738420941757

30. Shrivastava RM, Shrivastava L, Shrivastava R. Dual acting polymers in an osmotic film for topical application to treat inflammatory diseases and cytokine release syndrome. Patent PCT/EP2020/075117. EU. 2020

31. Singhal TA. Review of Coronavirus Disease-2019 (COVID-19). Indian J Pediatr. 2020;87:281-286. doi:10.1007/s12098-020-032636

32. Zhang L, Jackson CB, Mou H, et al. SARS-CoV-2 spike-protein D614G mutation increases virion spike density and infectivity. Nat Commun. 2020:11-6013.

33. Shrivastava R, Shrivastava R, Johansen B, Allaïn T. Antiinflammatory and antiviral osmotic polymeric film to treat Covid-19 early-stage infection. $J$ Inflamm Res. 2021;14:1195-1206. doi:10.2147/JIR.S306434

34. Li Q, Wu J, Nie J, et al. The impact of mutation in SARS-CoV-2 spike on viral infectivity and antigenicity. Cell. 2020;182:1284-1294. doi:10.1016/j.cell.2020.07.012
Open Access Journal of Clinical Trials

\section{Publish your work in this journal}

The Open Access Journal of Clinical Trials is an international, peerreviewed, open access journal publishing original research, reports, editorials, reviews and commentaries on all aspects of clinical trial design, management, legal, ethical and regulatory issues, case record form design, data collection, quality assurance and data auditing methodologies. The manuscript management system is completely online and includes a very quick and fair peer-review system, which is all easy to use. Visit http://www.dovepress.com/testimonials.php to read real quotes from published authors. 